\title{
Structural flexibility of carbon nanotubes
}

\author{
Sumio lijima \\ Fundamental Research Laboratories, NEC Corporation, 34 Miyukigaoka, Tsukuba, Ibaraki 305, Japan
}

Charles Brabec, Amitesh Maiti, and Jerzy Bernholc

Department of Physics, North Carolina State University, Raleigh, North Carolina 27695-8202

(Received 2 August 1995; accepted 11 October 1995)

\begin{abstract}
We report high resolution electron microscope (HREM) observations and atomistic simulations of the bending of single and multi-walled carbon nanotubes under mechanical duress. Single and multiple kinks are observed at high bending angles. Their occurrence is quantitatively explained by the simulations, which use a realistic many-body potential for the carbon atoms. We show that the bending is fully reversible up to very large bending angles, despite the occurrence of kinks and highly strained tube regions. This is due to the remarkable flexibility of the hexagonal network, which resists bond breaking and bond switching up to very high strain values. (C) 1996 American Institute of Physics. [S0021-9606(96)02003-0]
\end{abstract}

Recent arc discharge syntheses of multi-walled ${ }^{1-3}$ and single-walled ${ }^{4,5}$ graphitic nanotubes have triggered intense research efforts exploring their novel electronic, chemical and structural properties for possible technological applications. From a large number of experimental and theoretical investigations, it is evident that nanotubes hold substantial promise for use as superstrong fibers, composites, catalysts, molecular straws, and even as molecular switches. ${ }^{6-9}$ However, for almost any application, it is important that they have a large length to diameter ratio and a low fraction of structural defects. These conditions together imply that an almost pure graphitic lattice be maintained during growth or handling. However, even during careful handling of the soot containing the nanotubes, spatial fluctuations of mechanical forces are sufficient to cause deformations (bending, twisting, or flattening). In this Communication, we examine the deformation properties of single and multi-walled nanotubes bent to large angles. The experimental and theoretical results show that the tubes are remarkably flexible and provide an atomistic picture of the bending process. Surprisingly, our molecular dynamics simulations show that the bending is completely reversible up to angles in excess of $110^{\circ},{ }^{10}$ despite the formation of complex kink shapes.

We have examined two types of nanotubes with the electron microscope: (i) multi-walled tubes grown in a carbon arc plasma chamber ${ }^{1}$ with an outer diameter of typically 5-10 nm, and (ii) narrow single-walled tubes, grown in the same carbon arc with the addition of iron vapor as a catalyst. ${ }^{4}$ A closer examination of the nanotubes reveals two distinct types of bending: (a) associated with structural discontinuity introduced as growth faults that are usually caused by disclinations initiated by pentagonal or heptagonal rings, ${ }^{2}$ and (b) caused by an apparent mechanical deformation under forced confinement of the ends (see Fig.1). TEM images of the bending pattern of type (a) can be easily distinguished from that of type (b). Bending of type (a) has been previously identified in TEM images ${ }^{2}$ and has been investigated theoretically, ${ }^{11}$ while only a few images of low-angle bending of type (b) on thicker multi-walled tubes have been re- ported to date, without detailed structural analysis. ${ }^{12}$

Our HREM images and simulations show that both single and multi-walled tubes can be bent up to a certain critical angle (varying with the tube diameter) without any deformation, the only effects being a simple stretching of the outer side of the tube and a compression of the inner side. Beyond that angle a buckling pattern emerges, which manifests itself in HREM as a periodic disruption in the basal plane lattice images (spacing $0.34 \mathrm{~nm}$ ) of the inner side of the curved tube, which is under compression. This disruption is caused by a large deviation from the Bragg diffraction condition for the electron waves in microscope imaging, and thus implies a distortion of the cylindrical lattice. Irrespective of the number of shells or their diameters, we always see the initial formation of a single kink, followed by a multiple kink pattern upon further bending. The outer side of the tube does not show the buckling pattern because it is always stretched. As the number of nested shells increases (or the inside hollow diameter decreases), there is an increase in the number of periodic disruptions in the buckling pattern. Figures 1(a) and 1(b) shows TEM images of two single-walled tubes of diameters $0.8 \mathrm{~nm}$ and $1.2 \mathrm{~nm}$, respectively, that have developed single kinks in the middle. The shape of the 1.2 $\mathrm{nm}$ tube is quantitatively reproduced in the simulations (see Fig. 2 and below). Figure 1(c) shows an image of a multiwalled tube that has developed a single kink near its center. Upon further bending the same tube develops double kinks as shown in Fig. 1(d). Each kink has a structure of sharply curved lattice layers. The outer side of the tube in Fig. 1(d) is heavily stretched at the top of the double kink.

The deformations in both single-walled and multi-walled tubes are presumably introduced during specimen handling, such as collecting the soot from the arc chamber and transferring it to the TEM specimen grid for examination. The ends of the bent tubes are seemingly not free, but held at the bundles of tubes or catalytic particles in the arc discharge apparatus. Comparing the TEM images of Fig. 1 with those reported by Despres et al. ${ }^{12}$ in which the bent nanotubes were observed in specimens that were not dispersed in an 


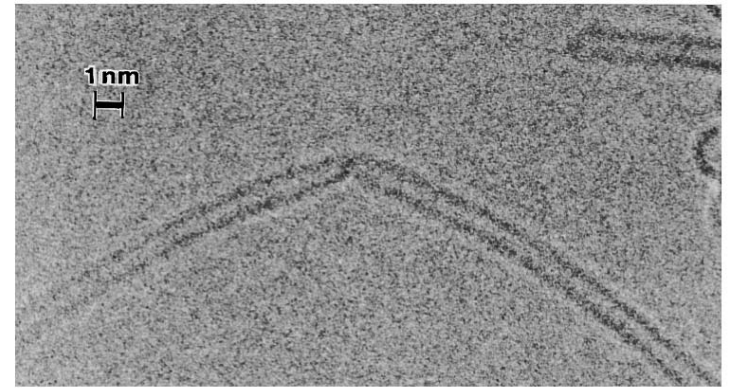

(a)

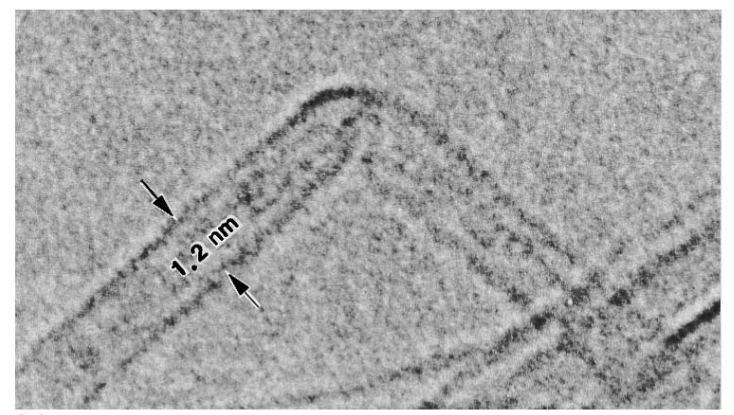

(b)

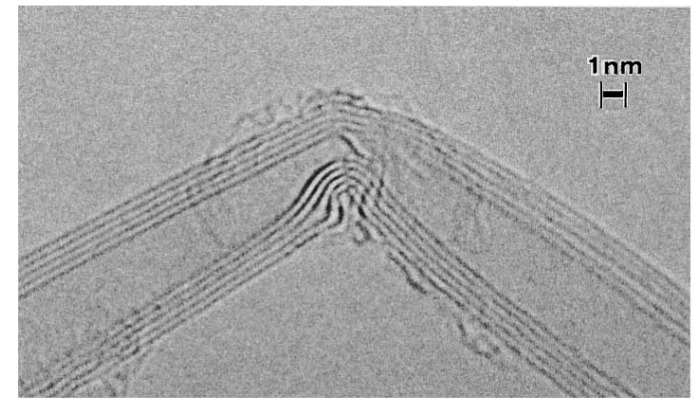

(c)

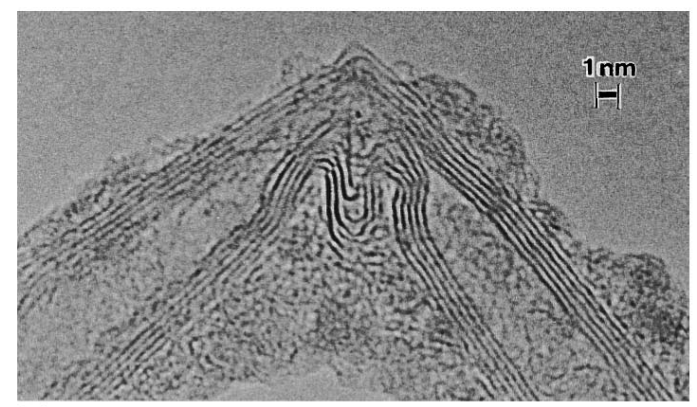

(d)

FIG. 1. HREM images of kink structures formed in nanotubes under mechanical duress. (a), (b) Single kinks in the middle of single-walled nanotubes with diameters of $0.8 \mathrm{~nm}$ and $1.2 \mathrm{~nm}$, respectively. The gap between the tip of the kink and the upper wall is about $0.4 \mathrm{~nm}$ in (b). (c), (d) A multi-walled tube (diameter $\sim 8 \mathrm{~nm}$ ) showing a single kink and a two-kink complex, respectively. The two kinks in (d) are separated by about $3.5 \mathrm{~nm}$.

ultrasonic bath, the conditions of our experiments appear to lead to much greater bending angles.

In order to reproduce the HREM images and to analyze bent structures in atomistic detail, we have performed computer simulations of bending of single and double-walled nanotubes. The interaction between the carbon atoms was modeled by the many-body potential due to Tersoff, ${ }^{13}$ as parameterized by Brenner. ${ }^{14}$ This potential is short-ranged, with

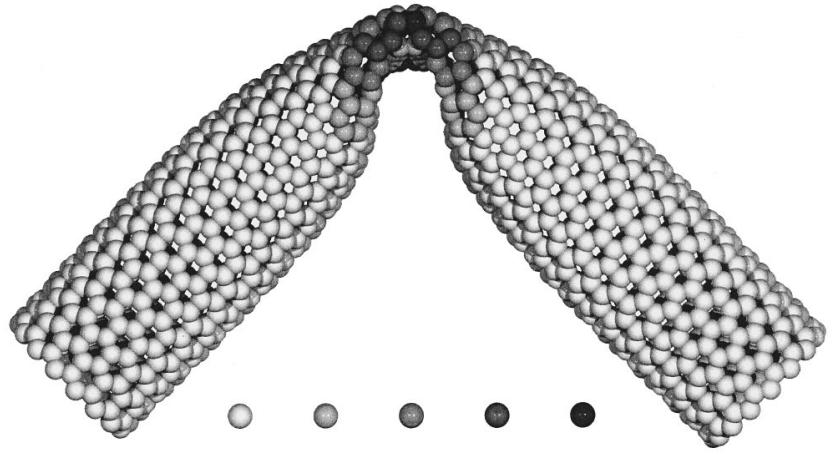

FIG. 2. Atomic structure of a single kink obtained in the computer simulation of bending of the single-walled tube in Fig. 2 (diameter $\sim 1.2 \mathrm{~nm}$ ). The coding denotes the local strain energy at the various atoms, measured relative to a relaxed atom in an infinite graphene sheet (Ref. 14). The strain energy scale ranges from 0 to $1.2 \mathrm{eV} /$ atom, from left to right.

a cut-off $<3 \AA$, and the total energy of the tube is written as a sum over the atomic sites in the local form

$$
E_{b}=\sum_{i} E_{i},
$$

where the difference between $E_{i}$ and the cohesive energy per atom of a flat, infinite graphene sheet ${ }^{14}$ can be interpreted as an excess "strain energy" localized at the site $i$ due to the local curvature of the bent tube. Each term $E_{i}$ depends on the pairwise distance of the neighbors relative to the atom $i$ and on the angles enclosed by the nearest neighbor triplets that include this atom. The parameters of this potential have been fitted to the experimental atomic densities and binding energies of the bulk phases of carbon, i.e., diamond and graphite, ${ }^{14}$ and have been used to study both the energetics ${ }^{15}$ and the growth properties ${ }^{16,17}$ of isolated nanotubes. Tersofftype potentials have also been used to study the deformation in nanotube bundles. ${ }^{18}$ In addition to the Tersoff-Brenner interaction, we have also included an additional van der Waals interaction ${ }^{19}$ between the adjacent shells of a multi-walled tube and between the top and the bottom sides of the same shell when they are in close proximity, e.g., just above a kink. The simulation for a tube of a given length, diameter and helicity was started by first relaxing the tube in the straight (horizontal) geometry. The bending angle was increased in small steps and the tube was relaxed at each position by the method of conjugate gradients. The presence of the bending force was simulated by keeping the end atoms fixed for each configuration. Up to 3000 atoms were included in the simulations.

We have carried out bending simulations with the above setup for a large number of single-walled tubes of varying diameters and helicities. In all simulations the same generic features appear: for bending below a sharp critical curvature, the tube undergoes a simple compression on the inner (bending) side, and a stretching on the outer side. This is the harmonic regime in which Hooke's law is obeyed, and the energy increases quadratically as a function of the bending angle, see Fig. 3. In the harmonic regime, the hexagonal rings on the tube surface are only slightly more strained than 


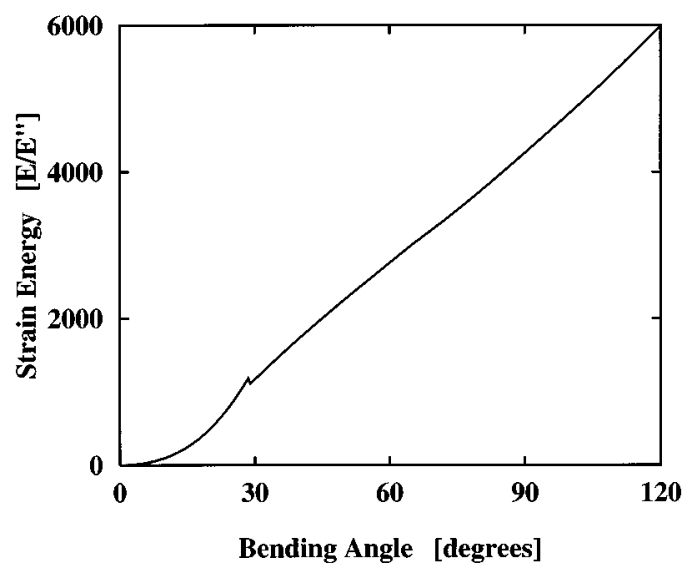

FIG. 3. The total strain energy (in dimensionless units) of a single-walled tube of diameter $\sim 1.2 \mathrm{~nm}$ as a function of the bending angle up to $120^{\circ}$. The energy is measured relative to the straight tube and the dip in the curve is associated with the formation of a kink. See text.

in a straight tube and they maintain an almost undistorted shape. At the critical curvature the strain on the compressed side reaches a maximum, and beyond this curvature the excess strain is released through the formation of a kink that increases the surface area of the bending side. This is accompanied by a dip in the energy vs bending angle curve, as shown in Fig. 3. Following the formation of the kink, the energy increases essentially linearly up to $\sim 120^{\circ}$. Figure 2 shows the structure of the kink in a single-walled tube of diameter $1.2 \mathrm{~nm}$. The overall shape of the kink, along with the distance of the tip of the kink from the upper wall of the tube is in quantitative agreement with the TEM picture of Fig. 1(b). This latter distance is about $0.35 \mathrm{~nm}$, precisely where the van der Waals interaction becomes strongly repulsive. ${ }^{19}$ The excellent agreement between the observed and the computed images confirms the reliability of our simulations, which provide complete atomistic and energetic information about the bending process. It is evident from the simulations that the perfect hexagonal network is preserved up to $\sim 110^{\circ}$, despite the occurrence of a kink above $\sim 30^{\circ}$. Only beyond a bending angle of $120^{\circ}$ bond-breaking occurs, leading to a damaged tube with dangling bonds upon unbending. In some very narrow tubes (diameter $\leqslant 1.0 \mathrm{~nm}$ ), we also find occasional bond-switching at bending angles $>110^{\circ}$, resulting in the formation of ring complexes involving pentagons and heptagons. However, below bending angles of $110^{\circ}$, no bonds are broken and no bond switching occurs, in spite of the high value of the strain energy in the exposed area (sides) of the kink, as hi-lighted by the color coding of Fig. 2. When the bending force is removed, the nanotube returns to its initial straight configuration without any hysteresis.

Extensive simulations of the bending of single-walled tubes with varying lengths, diameters and helicities show that the critical local curvature at which the kink forms is independent of the tube's length. It is well fitted by the equation

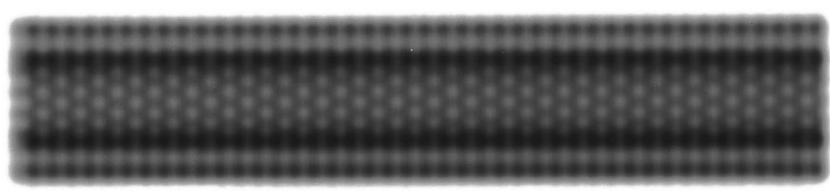

(a)

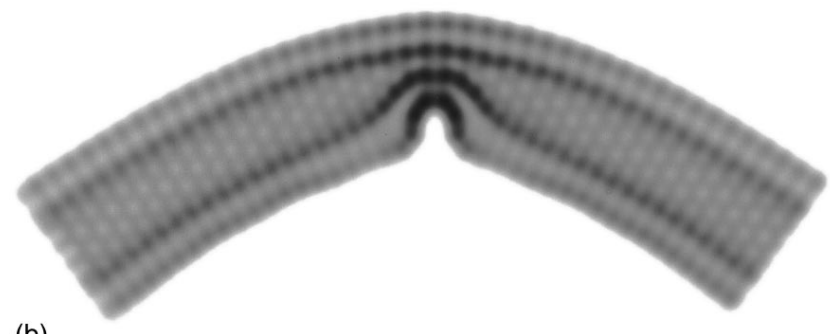

(b)

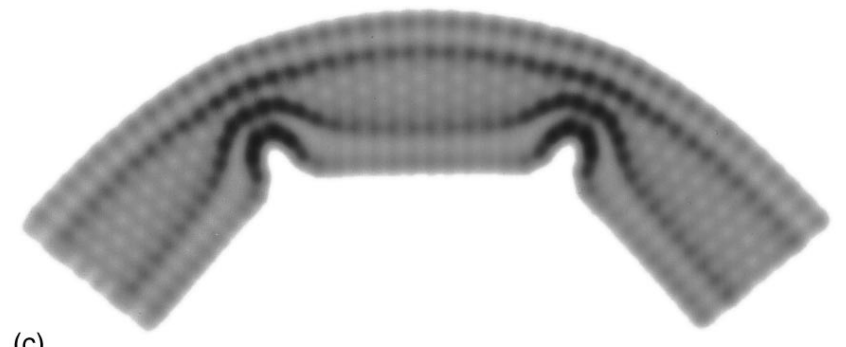

(c)

FIG. 4. Simulated cross-sectional images of a double-walled tube (diameters 1.0 and $1.7 \mathrm{~nm}$ ) under a progressively increased bending: (a) the straight relaxed tube; (b) a single kink forming above a critical curvature; and (c) a two-kink complex forming in the middle at large bending. The distance between the two kinks $(\sim 2 \mathrm{~nm})$ was found to be constant over a wide range of bend angles.

$$
C_{c}=\frac{1.49}{d^{2}}\left[1+\frac{9.89}{d^{5}} 10^{3} \cos (6 h)\right],
$$

where $C_{c}$ is the critical curvature in radians/ $\AA$ and $d$ is the tube diameter in $\AA$; $h$ is the tube's helicity, being 0 for a sawtooth tube, $\pi / 6$ for an armchair tube, and in-between for a general helical tube. The parameters of Eq. (2) have been fitted to bending simulations for single-walled tubes of diameters varying between $1.0 \mathrm{~nm}$ and $1.5 \mathrm{~nm}$ and covering the entire range of helicity $[0, \pi / 6]$. The helicity dependence is less than $1 \%$ for tubes wider than $2.0 \mathrm{~nm}$, and the critical curvature drops off as inverse square of the tube diameter, as expected from continuum elasticity theory. ${ }^{20}$ It is interesting to note that the fit given by equation (2) does not depend on the van der Waals interaction because, for single-walled tubes, the latter comes into play only after the kink has formed.

Encouraged by the remarkable agreement of Fig. 2 with the TEM picture of Fig. 1(b), we carried out bending simulations for a number double-walled tubes in order to qualitatively reproduce important features of the TEM images of the multi-walled tube in Figs. 1(c) and (d). The simulations for double-walled tubes lead to more complicated buckling patterns as a function of bending than for the single-walled tubes, as depicted in Fig. 4. Figure 4(a) shows a non-helical double-walled tube in its original relaxed state. Figure 4(b) 
displays a single kink in the middle of the tube. Just as in the single-walled tube, this kink forms at a sharp critical value of the local curvature. Upon further bending, there is a sharp transition from a single kink to a two-kink structure placed symmetrically about the middle of the tube [Fig. 4(c)]. The formation of a second kink is due to excess strain buildup because of the mismatch in compression on the bending sides of the two layers of the double-walled tube. Although two kinks formed both in experiment and in simulations, the agreement in the shape of the kink complex is not as good as in the case of the single tubes [cf. Figs. 4(c) and 1(d)]. This is mainly due to the presence of many more layers in Fig. 1(d). (A direct simulation of a 5-layer tube would be prohibitively expensive at present.) Further, the HREM image in Fig. 1(d) shows that the multi-layer tube has residual interactions with disordered graphitic layers, which also affects the quantitative comparison. However, the same principle must clearly be operating behind the formation of multiple kinks in a multi-walled tube as in the double-walled tube. In the simulation of the tube shown in Fig. 4, no noticeable change in the distance between the two kinks was observed when the bending was increased to as much as $110^{\circ}$. Furthermore, as in the single-walled tubes, no bond breaking or switching occurs before the bending angle becomes large $\left(>120^{\circ}\right)$, much greater than the angle corresponding to the formation of a kink or a kink complex. Thus, within a wide range of bending, the tube retains an all-hexagonal structure and reversibly returns to its initial straight geometry upon removal of the bending force.

In all simulations of both single and double-walled tubes, the first kink always forms in the middle of the tube where the maximum strain occurs. In the presence of the kink, the strain is always maximum at the atoms in the highly stretched region of the tube wall between the tip of the kink and the outer wall. The outer wall just above the kink is also highly strained, while the atoms lying on the kink are much more relaxed, see Fig. 2.

Simulations at room temperature show small amplitude ripples due to the formation of standing waves on the bend- ing side of the tube. This leads to kink formation at a slightly smaller bending angle than at zero temperature. However, just as in the zero temperature case, the tube retains its graphitic structure up to very large bending angles. Thus for a wide range of practical conditions, carbon nanotubes are very flexible during bending and show substantial promise for structural and fiber applications.

We thank B. I. Yakobson for valuable discussions.

${ }^{1}$ S. Iijima, Nature (London) 354, 56 (1991)

${ }^{2}$ S. Iijima, T. Ichihashi, and Y. Ando, Nature (London) 356, 776 (1992).

${ }^{3}$ T. W. Ebbesen and P. M. Ajayan, Nature (London) 358, 220 (1992).

${ }^{4}$ S. Iijima and T. Ichihashi, Nature (London) 363, 603 (1993).

${ }^{5}$ D. S. Bethune, C. H. Kiang, M. D. de Vries, G. Gorman, R. Savoy, J. Vasquez, and R. Bayers, Nature (London) 363, 605 (1993).

${ }^{6}$ P. Calvert, Nature (London) 357, 365 (1992).

${ }^{7}$ P. Ross, Sci. Am. 265, No. 6, 16 (1991).

${ }^{8}$ J. Broughton and M. Pederson, Phys. Rev. Lett. 69, 2689 (1992).

${ }^{9}$ N. Hamada, S. Sawada, and A. Oshiyama, Phys. Rev. Lett. 68, 1579 (1992); J. W. Mintmire, B. I. Dunlap, and C. T. White, ibid. 68, 631 (1992); R. Saito, M. Fujita, G. Dresselhaus, and M. S. Dresselhaus, Appl. Phys. Lett. 60, 2204 (1992)

${ }^{10} \mathrm{We}$ define the bending angle as the smaller angle between the tangents at the two ends of the bent tube. For a given level of local strain in the middle of the tube, this angle depends upon the tube length, and it is more appropriate to associate the deformation with the local curvature [see Eq. (2) in text]. However, to impart a more visual feeling, we often discuss bending conditions in terms of bending angles measured for a reference tube length of $9 \mathrm{~nm}$.

${ }^{11}$ B. I. Dunlap, Phys. Rev. B 46, 1933 (1992).

${ }^{12}$ J. F. Despres, E. Daguerre, and K. Lafdi, Carbon 33, 87 (1995).

${ }^{13}$ J. Tersoff, Phys. Rev. Lett. 56, 632 (1986); 61, 2879 (1988); Phys. Rev. B 37, 6991 (1988)

${ }^{14}$ D. W. Brenner, Mat. Res. Soc. Symp. Proc. 141, 59 (1989); Phys. Rev. B 42, 9458 (1990).

${ }^{15}$ D. H. Robertson, D. W. Brenner, and J. W. Mintmire, Phys. Rev. B 45, 12592 (1992).

${ }^{16}$ A. Maiti, C. J. Brabec, C. Roland, and J. Bernholc, Phys. Rev. Lett. 73, 2468 (1994)

${ }^{17}$ C. J. Brabec, A. Maiti, C. Roland, and J. Bernholc, Chem. Phys. Lett. 236, 150 (1995).

${ }^{18}$ J. Tersoff and R. S. Ruoff, Phys. Rev. Lett. 73, 676 (1994).

${ }^{19}$ H. A. Rafizadeh, Physica (Utrecht) 74, 135 (1974).

${ }^{20}$ B. I. Yakobson, C. J. Brabec, and J. Bernholc (unpublished). 